\title{
A Brief Analysis of the Moral Education in the Comprehensive Chinese Course for Foreign Students
}

\author{
Yining $\mathrm{Li}^{1, \mathrm{a}}$,Xinjun $\mathrm{Fu}^{2, \mathrm{~b}^{*}}$
}

\author{
${ }^{1}$ Department of Humanity and management, Shaanxi University of Chinese Medicine, Xianyang, Shaanxi, China \\ ${ }^{2}$ Department of Humanity and management, Shaanxi University of Chinese Medicine, Xianyang,Shaanxi, China \\ alyn361145656@qq.com \\ b*fuxinjun402@126.com
}

\begin{abstract}
In recent years, the number of overseas students studying in China is increasing, and the trend is increasing year by year. However, due to their different living habits, learning styles and other aspects, it is difficult for some overseas students to integrate into China's modern society, and there are conflicts with local students. In order to improve this kind of situation, this paper focuses on Chinese comprehensive course and briefly analyzes how to strengthen the moral education of foreign students by using the comprehensive course of Chinese as a foreign language.
\end{abstract}

Keywords: Chinese as a Foreign Language, abroad students, moral education, comprehensive course

\section{浅析留学生对外汉语综合课中的德育教育}

\author{
李伊宁 ${ }^{1,}$ a 付新军 $^{2,}$ b*
}

${ }^{1}$ 陕西中医药大学人文管理学院, 咸阳, 陕西, 中国

2 陕西中医药大学人文管理学院，咸阳，陕西，中国

alyn361145656@qq.com

$b^{*}$ fuxinjun402@126.com

\section{摘要}

近年来, 来中国求学的留学生数量日益增多, 且有逐年上升的趋势。但由于其生活习惯、学习方式等方面的不 同, 使得一些留学生难以融入中国现代社会, 且与当地学生时有矛盾发生。为了改善这类情况, 本文以汉语综 合课入手，并浅析如何利用对外汉语综合课加强对留学生的德育教育。

关键词：对外汉语，留学生，德育，综合课

\section{1. 前言}

随着中国国际地位的日益提高, 以及“中国力量” 在面对大型公共卫生事件时的出色表现，使得别国同 中国经济、文化等方面交流的渴望逐年增强, 来中国 求学的留学生数量也日益增多。来中国求学的留学生 中, 医学留学生和汉语留学生占了不小的比例, 且大 多数留学生都是选择高中毕业后来中国攻读大学, 其 三观还未完全定型。

近年来留学生与中国学生的摩擦时有发生, 甚至 有愈演愈烈的趋势。有几次大型冲突还曾霸榜新闻,
引起了社会各界广泛的关注和各地网友激烈的讨论。 由此可见, 加强对留学生德育的教育迫在眉睫。

对外汉语综合课作为对外汉语教学的核心课程 之一, 兼顾语言与文化, 能够更好的为加强德育教育 提供便于理解的背景并搭建好更加便于沟通的桥梁, 故本文选择对外汉语综合课, 浅析如何利用对外汉语 综合课加强对留学生的德育教育。 


\section{2. 对外汉语综合课}

对外汉语综合课是对外汉语课程中最为核心的 课程, 不同于专业技能课和单独的听力课、阅读课或 写作课, 综合课在初级、中级和高级这三个留学生的 汉语学习阶段, 都可以进行综合性的训练, 它能够将 听、说、读、写与文化相结合，关于其定义，在不同 时段不同的学者有着不同的说法:

吕必松 (1997) 认为综合训练就是要开设一门综 合课, 对语言知识、语用知识和文化知识以及言语技 能和言语交际技能进行教学和训练。

崔永华 (1999) 指出综合课的主要任务是对一个 教学单元的内容进行全面的引进, 其中便包含了语音、 词汇、语法点、文化点和课文等诸多要素, 并针对其 进行一定的语言技能训练。其同样强调了综合课处于 对外汉语教学主干课的地位, 并提出综合课的教学模 式是由二十世纪八十年代的对外汉语 “讲练--复练” 模式的讲练课发展而来的。

赵金铭（2004）对综合课进行了定义, 他认为综 合课是一门培养学生综合运用语言能力, 在课程设置 中处于基础和主干地位的课程, 其核心任务是语言技 能的训练。

李泉（2010）从课程的设置、层级、任务和模式 以及实际教学情况方面对汉语综合课的性质和特点 进行了深入的研究和探讨, 再次肯定和强调了综合课 的核心和主干地位, 明确了综合课的核心要务是 “培 养学习者的综合语言能力”。

杨惠元 (2010) 从提高课堂效率方面深入探讨了 与综合课相关的十种关系, 他认为为了提高综合课的 课堂效率, 应合理调节 “综合课与技能课” “语言要 素教学与语言技能” “听说与读写” 等多种关系, 教 师在教学实践中, 只有根据已有经验和实际情况处理 调节好这些在实践中所出现的关系, 才能不断提高教 学水平, 达到良好的课堂效果。

通过以上学者在不同时期对综合课性质定义及 其特点的探讨, 我们可以总结出对外汉语综合课是一 门涵盖了语音、词汇、语法, 并结合了文化着重培养 学生综合掌握听、说、读、写语言能力和训练其言语 技能的课程。

由此可见, 综合课结合了中文本身与其相关的文 化知识, 使其既能以合适的载体进行传播, 又能有恰 当的背景作为铺垫, 使留学生能够更好地在此背景下 理解并认可其蕴含的德育思想, 将二者巧妙地结合, 将会取得 “双赢” 的学习成果, 因此选择在综合课中 加强留学生的德育教育是很好的选择。

\section{3. 加强对留学生德育教育的必要性}

随着中国的综合实力进一步提升以及 “一带一路” 政策的顺利开展和国家的大力扶持, 中国的国际地位
逐渐攀升, 而结合从 2019 年至今, 中国在应对重大 公共卫生安全事件时在国内外的优异表现, 则更加体 现出了中国的 “大国担当”。近些年选择来中国求学 的留学生数量日益增加, 我们相信在国际后疫情时代 将会有越来越多的留学生会选择来中国留学。

无论外国留学生来到中国选择学习什么专业, 这 其中都应该包含德育的要求。“立德树人” 的要求将 同样适用在留学生的教学过程中。当然在面对的对象 不同时, 德育中 “德” 的具体含义可以有所区别, 但 其基本的要素则都应具备。我们之所以持续重视德育 方面的工作, 是因为我们注意到有一些发生在留学生 身上较为极端的事件, 如外籍学生与本地学生的冲突, 留学生擅闯女更衣室等, 它们从侧面反映出德育工作 的重要性。

对外汉语教学专家刘珣先生在 2008 年的一次报 告中就已经说过: “我们要用世界人民共同承认的一 些道德继续教育学生, 这样培养回国的人, 不仅学好 了汉语, 而且至少是一个很好的人, 品德各方面都很 好, 而且还懂得中国的一些文化, 遵守世界公德, 比 如遵守时间、爱护环境、爱护妇女等等, 这都是世界 每个国家都承认的公德, 这些在我们的课堂教学内容 中也要有, 让他回国以后有比较好的品质。”

因此, 在教学中我们要紧紧把握住对外汉语课程 的特点, 采用多样化教学, 把德育融入到课堂教学中, 把学生培养成全面发展的人才。

\section{1. 来华留学生现状分析}

中国学生的德育工作一般从小学就已经开始, 贯 彻整个学习生涯, 就语言能力来说, 大多数初到中国 留学生的中文能力跟小学生的中文能力较为相似, 所 以如果能从初级对外汉语综合课就开始进行留学生 的德育教育, 那将能使留学生从开始接触汉语时就能 接触德育, 以便于让他们更好的融入中国的社会, 适 应中国的生活方式, 遵守基本的行为规范, 也能让他 们更加理解中国人的行为准则, 这也能加快促进留学 生与中国学生、老师的良性交际。

大多数来中国求学的留学生都是选择高中毕业 来中国攻读大学, 虽然他们有一定的认知能力, 但其 三观尚未定型, 性格也还未定性, 且此时正处于完善 三观的关键期, 更需要外界对他们进行正向的引导, 带领他们树立正确的人生观、世界观和价值观, 这也 能让他们更好地融入中国现有的社会价值体系, 避免 由此引发一些有违公德的出格行为。与此同时, 引导 他们参与良性的社交活动, 保障其良好的身体和心理 素质, 提高抗压能力, 同时也能加速他们的跨文化的 适应进程, 促使其自觉提升自身素养和规范自身行为。

其次, 来华留学生的背景也不同, 他们大多有着 不同的社会文化、习俗和宗教文化背景。因此, 如果 不能有效地加强其教学管理, 那么教学效果就会出现 很大的差异, 会影响我们的教学进度, 为了使留学生 
能够学有所成, 摒弃 “镀金” 思想, 加强留学生的德 育教育势在必行。

\section{2. 来华留学生专业现状分析}

据数据统计, 来华留学生主要集中在医学和汉语 两个专业。以医学为例, 中医是中国独有的特色专业, 而留学生中大多数都是高中毕业生, 因此他们在此之 前可能从未接触过中医的内容, 无论学习什么专业, 显然汉语的学习都是一个必须的前提, 对于中医的学 习来说, 这方面的要求就需要更好, 因为他们对汉语 的需求将不止于现代汉语, 还需要掌握繁体字、古汉 语和各种带有哲学化含义的名词术语。因此对外汉语 综合课的开设是十分必要的, 而在综合课中对留学生 进行德育的教学设计, 显然是一个很好的选择而如果 能在他们求知欲望十分强烈的时期, 给他们奠定良好 的道德基础, 无疑会在他们以后的从医道路上受益无 穷。（郑晓玲 2001）而如果留学生的德育教育工作长 期处于空白阶段, 或是对其无法真正的赞同和有所感 悟, 那么他们学到的就只是业务技术知识, 而医者身 上极为重要的灵魂要素 “医德” 就会不完备, 甚至缺 失。中医学始终将个体的人作为诊治的对象, 强调的 天人合一的整体观, 这其中尤其能体现出中医学以人 为本的 “仁学” 特点, 所谓 “仁” 即指爱人, 所以孙 思㳶提出医者要有佛家的大慈恻隐之心, 又要行儒家 的忠恕之道。这种情况不能真正体现出中医的内涵, 且也会影响到他们未来的职业成就。

既然他们已经选择来华学习中医知识, 那我们将 不能仅仅满足于教会他们 “如何” 行医, 也该让他们 对 “为何” 行医有所体会, 更有必要让其感受到孕育 中国医药学这一伟大民族的深厚道德底蕴, 弘扬中华 传统文化, 这就是进行德育工作的重要性和必要性。

同理, 对于选择其他学科求学的留学生, 也可将 对外汉语综合课结合其学科的相关背景, 加强对留学 生的德育教育。

\section{4. 加强留学生综合课的德育教育}

留学生来华不仅要学习专业知识和技能, 还要学 习汉语, 因此汉语综合课的学习必不可少, 利用汉语 综合课综合性的特点, 和课堂渠道的便捷性, 也能够 更好地进行留学生德育的教育工作。

\section{1. 结合综合课内容加强德育教育}

以 HSK1 第四课 “她是我的汉语老师” 为例, 在 介绍老师和朋友的同时，也可以加入中国“尊师重教” 的传统文化进行德育教育。还可以在小组提问的环节, 去提问来自不同国家的同学, 以了解他们国家学生跟 老师的关系是怎么样的, 是师生平等还是老师位居上 位, 讨论他们认为老师的职责是什么, 再进行交流。 以此普及我们现今社会的师生关系, 以及尊师重教的 传统。此外适当的介绍一下孔子的教学思想, 也有助
于教学工作和德育工作的进行。比如粗略地讲解一下 “因材施教”和 “有教无类” 的故事, 但教学内容的 层次不必太深入，因为深入教学将使 HSK1 的学生难 以理解, 所以在进行交流讨论的时候也要注意, 使其 适当和适度地进行。

另外, 在综合课中还有很多交际情景可以融入课 堂中, 在交际情景中进行的对话, 我们可以试着以带 有德育的背景进行设置，例如以下对话：

（过马路要遵守交通规则）

汤姆: “李明, 你要去哪儿啊? ”

李明：“我去书店买书，你呢？”

汤姆: “我也去书店买书, 我们一起吧。你怎么 不过马路啊？”

李明: “现在是红灯, 绿灯还有三十秒, 绿灯才 能过马路。”

汤姆: “那我们一起等绿灯吧。”

将德育情景加入交际训练或者课堂对话中, 能让 他们更好的理解和进行运用。

诸如此类, 如果有必要还可以分阶段单独开设讨 论课, 让留学生能够在讨论中了解并理解中国文化以 及现阶段中国的社会情况，另外还可以从正反两个方 面对一些典型的中外社会中出现的情景进行是非、真 假、善恶、美丑的剖析和思辨, 加强留学生语言运用 能力的同时，产生积极的榜样示范与耻感效应。

\section{2. 开展校园活动补充进行德育教育}

此外, 还可以结合定期开展各种校园活动如体育 比赛、文艺演出、汉语知识竞赛、汉语主题演讲比赛、 汉语辩论赛等, 鼓励留学生多多参与, 以推动留学生 之间的交往、加强师生之间的沟通、增进中外学生的 了解, 达到消除隔阂、增进友谊的良好循环，以这种 潜移默化的方式补充进行德育教育, 最终实现留学生 思想道德素质的提升。

结合留学生的专业来看, 如医学院校的留学生在 学习到一定阶段, 可以进入实践之后, 还可以跟随专 业院系的学生去进行义诊, 在提升实践能力的同时, 还可以让他们切身体会到医德品质和奉献精神在生 活中的体现。

\section{3. 开展课外活动加强德育教育}

另外, 还可以适时开展一些课外活动, 比如组织 留学生汶览当地的景点体验其自然和文化风光, 或是 让他们去超市、商场等场所锻炼语言运用能力，观察 在此场景下中国人的生活方式和行为准则, 同时也鼓 励留学生适当地多交一些中国朋友，观察中国朋友是 怎样跟家人、长辈、同学、朋友相处的, 让留学生能 够切身体会到感受中国其乐融融的家庭氛围以及在 
中国社会中波源流长的传统美德。

最后, 还可以鼓励留学生多参加社会实践活动, 比如马拉松比赛、大运会、音乐节等的志愿者, 让留 学生在参与社会志愿活动中逐渐取得社会认同感, 还 可以参加社区活动, 比如给养老院、留守儿童献爱心, 让留学生体会中华民族团结友爱的精神。

\section{5. 结语}

综上所述, 利用好对外汉语综合课, 结合一些必 要的校园活动与社会活动, 以加强对留学生的德育教 育工作, 将会起到事半功倍的效果。作为国际中文教 师, 这也是我们要多进行思考的方向, 不能仅仅局限 于教好中文, 还应该适当地进行拓展, 让留学生 “理 解” 中文, 帮助他们更好地融入中国现代社会, 因为 我们是他们的 “领路人” , 更是他们与中文乃至中国 之间的桥梁, 因此德育教育, 对于我们国际中文教育 者们来说责无旁贷。

\section{REFERENCES}

[1] Liu,Y.(2021) The infiltration of moral education in the Comprehensive Practice activity of Primary school [A]. The Research Institute of Education Science of Chinese Academy of Management Science.2021 Education Innovation Webinar proceedings (I) [C]. Institute of Education Science, Chinese Academy of Management Sciences: Institute of Education Science, Chinese
Academy of Management Sciences, 2021:2.

[2] Lu,T,H.(2019)Three-dimensional Path of Moral Education Construction for International Students in Universities [J]. Research of Ideological Education,(10):130-133.

[3] Zheng,X,L.(2001)On The Moral Education of Foreign Students in Chinese Medicine Colleges [J]. Chinese Medicine Education,(16):26-27.

[4] wang,F,L.(2012)Discussion on moral education penetration in teaching Chinese as a foreign language $[\mathrm{J}]$. Journal of shenyang university (social science edition),14(02):73-75.

[5] Lv,P.(2010)Using Tao Xingzhi's Moral Education Thought to guide the Moral Education of International Students in China [J]. China Afterschool Education,(14):2.

[6] Wang,C,G.(2012)The Necessity and Ways of Strengthening ideological and moral Education of International Students [J]. Journal of tonghua normal university,33(01):93-96.

[7] Zhou,L.(2016)Analysis on Management Mode of International Students in Colleges and Universities and Research on Countermeasures of Moral Education [J]. Management and Technology of Small and Medium-sized Enterprises (Midday),(12):149-150. 